Research Article

\title{
The Research on Huanglian Jiedu Decoction against Atopic Dermatitis
}

\author{
Yu-Bin Xu \\ Taizhou Central Hospital, Taizhou University Hospital, Taizhou, Zhejiang 318000, China \\ Correspondence should be addressed to Yu-Bin Xu; xuyubin1988@126.com
}

Received 27 January 2021; Accepted 13 February 2021; Published 23 February 2021

Academic Editor: Wenzheng Bao

Copyright (C) $2021 \mathrm{Yu}$-Bin Xu. This is an open access article distributed under the Creative Commons Attribution License, which permits unrestricted use, distribution, and reproduction in any medium, provided the original work is properly cited.

Objective. Study on the pharmacodynamic basis and mechanism of Huanglian Jiedu Decoction against atopic dermatitis (AD). Methods. Based on network pharmacology, the targets of Huanglian Jiedu Decoction and AD were screened by Traditional Chinese Medicine Systems Pharmacology Database and Analysis Platform (TCMSP), SwissTargetPrediction databases, and the database of Online Mendelian Inheritance in Man (OMIM), Therapeutic Targets Database (TTD) and the Comparative Toxicogenomics Database (CTD); then, "chemical composition-target-related pathway-disease target" network graph of Huanglian Jiedu Decoction against AD was constructed by using STRING and Cytoscape software. In combination with in vitro experiments, the levels of IL-4, IL-6, and IL-10 in T cells were determined by ELISA; the pharmacodynamic basis and mechanism of Huanglian Jiedu Decoction against AD were preliminarily explored. Results. 81 active ingredients in Huanglian Jiedu Decoction were screened by network pharmacology, 31 of which were related to atopic dermatitis, corresponding to 12 target proteins. A total of 14 pathways were obtained by KEGG pathway analysis, and 8 were associated with atopic dermatitis. Compared with the control group, 20 and $40 \mu \mathrm{g} / \mathrm{ml}$ of Huanglian Jiedu Decoction could significantly reduce the contents of IL-4, IL-6, and IL-10 in T lymphocytes of mice with atopic dermatitis $(p<0.01)$. Conclusion. Huanglian Jiedu Decoction can act against AD by multicomponent, multitarget, and multichannel mode of action.

\section{Introduction}

Atopic dermatitis is a kind of recurrent dermatitis caused by IgE mediate; it usually occurs during one's childhood, and it has characteristic age-dependent distribution; by statistics, the morbidity of this disease is $2-10 \%$ and as high as $15-30 \%$ for adult and children, respectively $[1,2]$. The pathogenesis is complex, and it contains many factors; it is generally recognized that the abnormality of immune function is the core of pathogenesis of atopic dermatitis [3, 4]. Its clinic presentation includes dry skin and severe pruritus with some other diseases such as anaphylactic rhinitis and asthma $[1,5]$. The research in recent years shows that atopic dermatitis relates to upregulation of type- 2 immune response. T helper cells (Th2) will generate interleukins IL-4, IL-6, and IL-10, and it will activate eosinophils and mastocyte and generate $\mathrm{B}$ cells for immune globulin $\mathrm{E}$ (IgE) and release histamine, interleukins IL-4, IL-5, IL-10, IL-12, and IL-13, as well as cytokines and other medium $[2,4]$; such medium can cause tissue injury and skin pruritus and make the inflammatory response procedure more serious. Besides, the research of Chun-Kwok et al. [6] also proved that interleukins IL-31 (cytokine of pruritus) and IL-33 (alarming for destructive inflammation) can reinforce the stimulus of released cytokines caused by eosinophils and fibroblast and chemokines relating to atopic dermatitis.

The serious atopic dermatitis will affect the living quality of the patient and family members, and proper treatment can release pruritus, sleeping dysfunction, infection, behavioral problems, and other symptoms [7]. Glucocorticoid, calcineurin inhibitor, and histamine-containing drugs are usually used to treat atopic dermatitis clinically, and an immunosuppressor is used to treat the patients with serious atopic dermatitis; however, taking glucocorticoid and calcineurin inhibitor for long time will cause some adverse reactions such as adermotrophia, coarse pore, pruritus, burning sensation, and tachyphylaxis. Besides, the "phobia for corticosteroid" and high expenditure for long-term 
treatment against atopic dermatitis occurred in the patients in recent years, and such will cause bad obedience of patients $[8,9]$. While because Chinese herb medicine has relatively lower toxicity, it has been accepted by more and more patients recently.

Huanglian Jiedu Decoction is one of the compounds of Chinese herb treating atopic dermatitis $[10,11]$, and it is composed of four kinds of Chinese herb of Coptis chinensis, Scutellaria baicalensis, golden cypress, and jasmine according to the proportion of $3: 2: 2: 3$; it has the function of purging fire for removing toxin. The modern pharmacological research proves that Huanglian Jiedu Decoction has the pharmacological effects of anti-inflammation, anticerebral ischemia, antitumor, immunoregulation, as well as antidiabetics and lipid-lowering; clinically, it is used to treat many diseases such as stroke, hypertension, diabetes, sepsis, and atopic dermatitis $[12,13]$.

Network pharmacology is based on the network of "ingredient-target spot-pathway-disease;" it can analyze the effect of medicines to disease comprehensively and discuss the action mechanism of medicine. Network pharmacology can not only analyze the mechanism of medicine of mono-compound but can also discuss the effect and potential mechanism of medicine combination, and it achieves the same result of traditional theory of Chinese medicine by different methods [14-16]. In this research, the method of network pharmacology is used to research the affecting mechanism of active ingredients of Huanglian Jiedu Decoction in treating atopic dermatitis, and the multipoint relativity effect network of "chemical ingredient-target spot-relevant pathways-target spot of disease" is determined to provide theoretical rationale for further research of verification and experiments.

\section{Materials}

10 SPF-level C57BL/ 6 female rates, $20 \pm 2$ g, were purchased from Beijing Charles River Experiment Animal Technology Co., Ltd.; the number of certificate of inspection is SCXK (J) 2012-2011.

2.1. Main Reagents. RPMI-1640 medium is purchased from Hyclone Company, bovine serum is purchased from Gibco Company (lot number: 8166872), double antibody is purchased from Hyclone Company (lot number: J120721), and IL-4, IL-6, and IL-10 reagent boxes (lot number: 201807) were purchased from Nanjing Jiancheng Bioengineering Institute (Nanjing, China).

2.2. Main Instruments and Equipment. Sartorious CP225D precision balance (Beijing Saiduosi Instrument System Co., Ltd.), Eppendorf adjustable pipette, TDZ4-WS low-speed desk centrifuge (Changsha Xiangzhi Centrifuge Instrument Co., Ltd.), HC-2518R high-speed freezing centrifuge (Anhui Zhongke Zhongjia Science Instrument Co., Ltd.), and microplate reader MULTISKAN FC (Thermo).

\section{Methods}

\subsection{Network Analysis}

3.1.1. Research Thought. For the sketch map on the thought of network pharmacological experiment of treating atopic dermatitis with Huanglian Jiedu Decoction.

3.1.2. The Selection of Active Ingredients. Search Traditional Chinese Medicine Systems Pharmacology Database and Analysis Platform [17] (TCMSP, http://lsp.nwu.edu.cn/ tcmsp.php) with Coptis chinensis, Scutellaria baicalensis, golden cypress, and jasmine (the main ingredients of Huanglian Jiedu Decoction) as the keywords to mine the data of current ingredients of Huanglian Jiedu Decoction, the screening according to ADME features of Chinese medicine, the utilization degree of oral taking and digesting $(>30 \%)$, and drug similarity ( $\mathrm{DL}>0.18)$; the achieved results are the active ingredients.

3.1.3. The Reverse Prediction of the Potential Effects and Target Spot of Active Ingredients. Log in SwissTargetPrediction [18] (http://www.swisstargetprediction. $\mathrm{ch} /$ ) and upload SDF/MOL file on the active ingredients of Huanglian Jiedu Decoction to obtain the virtual screening results with the method of inverse pharmacophore matching. Search potential medicine target with active small molecules as a probe to predict the biological activity of the compounds.

3.1.4. The Screening of the Affecting Target for Active Ingredients. Compare and analyze the information on the relevant targets of atopic dermatitis reported in the databases of Online Mendelian Inheritance in Man (OMIM) (http://www.omim.org/), Therapeutic Targets Database (TTD) (http://bidd.nus.edu.sg/group/cjttd/), and the Comparative Toxicogenomics Database (CTD) (http:// ctdbase.org) [19] and summarize the target spot genes shared by the both.

3.1.5. The Analysis on the Notes of the Target Spot Pathway. Perform KEGG (http://www.genome.jp/kegg/) [20] pathway enrichment analysis to the critical information on target spot via STRING and select the items with statistical significance $(p<0.01)$ to construct the network diagram of the "chemical ingredient-critical target spot-relevant pathway."

3.1.6. Constructing the Network Diagram of "Chemical Ingredient-Affecting Target Spot-Relevant Pathway-Target Spot of Disease". Map the relevant network information among ingredients, target spot, pathway, and diseases with Cytoscape software to investigate and research the affecting mechanism of Huanglian Jiedu Decoction toward atopic dermatitis. 


\subsection{Experiment Analysis}

3.2.1. Preparing for Huanglian Jiedu Decoction. First, we prepared $400 \mathrm{~g}$ Chinese medicinal materials according to the formula of Huanglian Jiedu Decoction. We began by soaking the medicinal materials in $4 \mathrm{~L}$ water for 30 minutes, which was then boiled for 60 minutes. They were filtered after boiling, and the filtrate was collected. A $3.2 \mathrm{~L}$ of water was added to the herbs mixture, boiled for $60 \mathrm{~min}$, and the filtrate was collected once again. The filtrates were combined, and the water was evaporated to obtain $4 \mathrm{~L}$ of concentrated liquid. This concentrated liquid was freeze-dried to finally get $40 \mathrm{~g}$ freeze-dried powder of HJD.

3.2.2. Feeding of Animals for Experiment. SPF-level C57BL/6 rats are fed in proper environment for 5 days. All animal procedures were approved by Liaoning Provincial Animal Welfare and Care Guidelines in accordance with the National Institute of Health's guidelines regarding the principles of animal care (2004).

3.2.3. Preparation of Lymphocyte. Take the eyeballs of C57BL/6 rats of the model of atopic dermatitis with success molding and killing of the rats; the model establishment can be referred to [21]; sterilize with $75 \%$ alcohol and soak them for 1-2 minutes; take the spleen out from super-clean workbench. Clean the spleen with sterilized saline (or d-hanks solution or PBS buffer solution) for three times. Clean away the fat and connective tissues on the surface of the spleen with tweezers and scissors, place them in the sterilized culture dishes with D-type handkerchief, cut the organs, and screen the cells on 200 meshes; at the same time, clean with D-Hanks solution and collect cell suspension. Separate $\mathrm{T}$ cells with nylon columns and the method are given in [22].

3.2.4. Culture of Lymphocyte. Prepare the solution of Huanglian Jiedu Decoction of $10 \mathrm{mg} / \mathrm{ml}$ before experiment, and measure and take dry paste of Huanglian Jiedu Decoction of $0.01 \mathrm{~g}$ and place it in the sterilized centrifuge tube of $1.5 \mathrm{ml}$; the tube should be placed in the super-clean bench after being sprayed with alcohol on its external wall; add $1 \mathrm{ml}$ of PRMI-1640 culture medium and mix evenly; then, take $100 \mu \mathrm{l}$ of the solution to place it in another centrifuge tube of $1.5 \mathrm{ml}$; then, add $900 \mu \mathrm{l}$ RPMI-1640 culture medium, and the concentration at this time is $1 \mathrm{mg} / \mathrm{ml}$; such solution is reserved for further use; take $100 \mu \mathrm{l}$ of solution above and add it to another centrifuge tube to prepare Huanglian Jiedu Decoction solution of $100 \mu \mathrm{g} / \mathrm{ml}$ for further use. Culture the purified $\mathrm{T}$ lymphocyte and then set the concentration of Huanglian Jiedu Decoction with different gradients of 0,10 , 20 , and $40 \mu \mathrm{g} / \mathrm{ml}$. Culture with a 24 -hole plate, and each batch will be processed for three times; add $1 \times 10^{5}$ cell of $1.5 \mathrm{ml}$ in each hole and intervene for $24 \mathrm{~h}$; then, collect cell supernatant using the ELISA method $[23,24]$ to test the content of IL-4, IL-6, and IL-10.

\section{Results}

\subsection{Network Pharmacology}

4.1.1. Screening of the Active Ingredients of Huanglian Jiedu Decoction. We have retrieved that there are 429 reported ingredients in Huanglian Jiedu Decoction via the TCMSP database, and with "OB $\geq 30 \%$ " and " $\mathrm{DL} \geq 0.18$ " and other ADME parameters as conditions, we have obtained 14 Coptis chinensis compounds, 32 Scutellaria baicalensis compounds, 37 golden cypress compounds, and 15 jasmine compounds after screening, in which there are 7 compounds shared by Coptis chinensis and gold cypress, 1 compound shared by Coptis chinensis and Scutellaria baicalensis, 1 compound shared by Scutellaria baicalensis and jasmine, 1 compound shared by Coptis chinensis, Scutellaria baicalensis, and Jasmine, 1 compound shared by Coptis chinensis, Scutellaria baicalensis, and golden cypress, and 2 compounds shared by Scutellaria baicalensis, golden cypress, and jasmine. There are 81 active ingredients in blood, as is seen in Supplementary information.

4.1.2. The Information on Potential Target Spot of Active Ingredients of Huanglian Jiedu Decoction. By the experiment of inverse molecular docking performed on SwissTargetPrediction service, it is predicted that 31 out of 81 active ingredients are relative to atopic dermatitis, and they correspond to 12 target protein; the total frequency is 48 . The general feature analysis to the "compound-target spot" network shows that, among 31 active ingredients, there is not only the fact that one compound is connected to many protein targets but also the fact that many compounds affect one target protein, and such facts present the feature of multiactive ingredients and multitargets of Huanglian Jiedu Decoction. After analyzing 12 target proteins, (1) the proteins relating to bacteria respond adjustment include CNR1, CNR2, MAPK8, NR1H3, and STAT1; (2) the proteins relating to inflammation adjustment include CNR1, CNR2, ELANE, NR1H3, and PLA2G2F; (3) the proteins relating to adjustment for external stimulus include ALOX5, CNR1, CNR2, MAPK8, NR1H3, PPARA, and STAT1; (4) the proteins relating to adjustment for endogenous stimulus include NR1H3, NR3C1, PPARA, STAT1, TRPV1, and PRSS1; and (5) the protein relating to immune adjustment include CNR1, CNR2, ELANE, MAPK8, NR1H3, and STAT1. We can herein predict that Huanglian Jiedu Decoction might be combined with the proteins relating to bacteria respond adjustment, anti-inflammation, endogenous and exogenous stimulus adjustment, as well as immune to treat atopic dermatitis, as shown in Table 1.

4.1.3. The Notes and Analysis of Affecting Pathways of Potential Target Spots. After KEGG pathway analysis made to the predicted target protein via STRING, there are 14 affecting pathways, in which 8 of them relate to atopic dermatitis: (1) 6 pathways relating to bacteria respond adjustment include neuroactive ligand-receptor interaction, influenza A, PPAR signaling pathway, adipocytokine 
TABle 1: Information of 12 target proteins in Huanglian Jiedu Decoction for the treatment of atopic dermatitis.

\begin{tabular}{|c|c|c|c|}
\hline No. & $\begin{array}{l}\text { UniProt } \\
\text { ID }\end{array}$ & Information of target proteins & The number combined with the active ingredient \\
\hline 1 & Q9BZM2 & Group IIF secretory phospholipase A2, PLA2G2F & 1 \\
\hline 2 & Q8NER1 & $\begin{array}{l}\text { Transient receptor potential cation channel subfamily V member } 1 \text {, } \\
\text { TRPV1 }\end{array}$ & 3 \\
\hline 3 & Q13133 & Oxysterols receptor LXR-alpha, NR1H3 & 9 \\
\hline 4 & Q07869 & Peroxisome proliferator-activated receptor-alpha, PPARA & 2 \\
\hline 5 & P45983 & Mitogen-activated protein kinase 8, MAPK8 & 1 \\
\hline 6 & P42224 & $\begin{array}{c}\text { Signal transducer and activator of transcription 1-alpha/beta, } \\
\text { STAT1 }\end{array}$ & 1 \\
\hline 7 & P34972 & Cannabinoid receptor 2, CNR2 & 5 \\
\hline 8 & P21554 & Cannabinoid receptor 1, CNR1 & 7 \\
\hline 9 & P09917 & Arachidonate 5-lipoxygenase, ALOX5 & 13 \\
\hline 10 & P08246 & Neutrophil elastase, ELANE & 1 \\
\hline 11 & P07477 & Trypsin-1, PRSS1 & 1 \\
\hline 12 & P04150 & Glucocorticoid receptor, NR3C1 & 4 \\
\hline
\end{tabular}

signaling pathway, pancreatic secretion, and Toll-like receptor signaling pathway; (2) 6 pathways relating to inflammation adjustment include neuroactive ligand-receptor interaction, arachidonic acid metabolism, PPAR signaling pathway, adipocytokine signaling pathway, retrograde endocannabinoid signaling, and Toll-like receptor signaling pathway; (3) 7 pathways relating to exogenous stimulus adjustment include neuroactive ligand-receptor interaction, influenza $\mathrm{A}$, arachidonic acid metabolism, PPAR signaling pathway, adipocytokine signaling pathway, pancreatic secretion, and Toll-like receptor signaling pathway; (4) 6 pathways relating to endogenous stimulus adjustment include neuroactive ligand-receptor interaction, influenza A, PPAR signaling pathway, adipocytokine signaling pathway, pancreatic secretion, retrograde endocannabinoid signaling; and (5) 7 pathways relating to immune adjustment include the neuroactive ligand-receptor interaction, influenza A, arachidonic acid metabolism, PPAR signaling pathway, adipocytokine signaling pathway, pancreatic secretion and Toll-like receptor signaling pathway. The concrete information is given in Table 2.

4.1.4. Constructing the Network Diagram of "Chemical Ingredient-Affecting Target-Relevant Pathway-Disease Target". The network diagram of Huanglian Jiedu Decoction of "compound-target-pathway-disease" has been established with Cytoscape software, and it has presented the relationship network between 31 compounds, 12 target proteins, 8 affecting pathways, and 5 kinds of affecting routine and atopic dermatitis. The more connecting lines between compounds and predicted protein targets, the larger the linear density will be, and it means that they tend to have more frequent interaction. In Figure 1, the light purple inverted triangle represents 31 kinds of compounds, rose triangle represents 12 target proteins, purple circle represents 8 affecting pathways, and green circle represents 5 affecting routines. CA1 means hericenone $\mathrm{H}, \mathrm{CA} 2$ means supraene, CA3 means crocetin, CA4 means melianone, CA5 means dihydroniloticin, CA6 means DIOP, CA7 means
TABLE 2: KEGG pathway derived from target protein analysis.

\begin{tabular}{lccc}
\hline No. & Pathway & Number & $P$ \\
\hline 1 & Neuroactive ligand-receptor interaction & 5 & 0.00191 \\
2 & Influenza A & 3 & 0.00628 \\
3 & Arachidonic acid metabolism & 3 & 0.0208 \\
4 & PPAR signaling pathway & 2 & 0.0208 \\
5 & Adipocytokine signaling pathway & 2 & 0.0208 \\
6 & Pancreatic secretion & 2 & 0.032 \\
7 & Retrograde endocannabinoid signaling & 2 & 0.0334 \\
8 & Toll-like receptor signaling pathway & 2 & 0.0341 \\
\hline
\end{tabular}

kihadalactone A, CA8 means mandenol, CA9 means palmidin A, CA10 means Candletoxin A, CA11 means GBGB, CA12 means beta-sitosterol, CA13 means campesterol, CA14 means delta-7-stigmastenol, CA15 means niloticin, CA16 means phellochin, CA17 means poriferast-5-en-3beta-ol, CA18 means stigmasterol, CA19 means 11,13eicosadienoic acid, methyl ester, CA20 means ethyl oleate (NF), CA21 means 5, $2^{\prime}, 6^{\prime}$-trihydroxy-7,8-dimethoxyflavone, CA22 means 5,8,2'-trihydroxy-7-methoxyflavone, CA23 means 5-hydroxy-7-methoxy-2-(3,4,5-trimethoxyphenyl) chromone, CA24 means baicalein, CA25 means isoimperatorin, CA26 means kaempferol, CA27 means phellopterin, CA28 means quercetin, CA29 means salvigenin, CA30 means wogonin, and CA31 means hispidone. From the figure, we can see that Huanglian Jiedu Decoction can treat atopic dermatitis with multiingredients, multitargets, and multipathways.

\subsection{Experiment Results}

4.2.1. Measurement of Inflammatory Factors. Comparing with the control group, Huanglian Jiedu Decoction of $20 \mu \mathrm{g} /$ $\mathrm{ml}$ and $40 \mu \mathrm{g} / \mathrm{ml}$ can obviously reduce the content of IL-4, IL-6. and IL-10 in T lymphocytes of rate in the atopic dermatitis mode $(p<0.01)$, and it indicates that Huanglian Jiedu Decoction at this concentration has the function of treating atopic dermatitis, as shown in Table 3. 


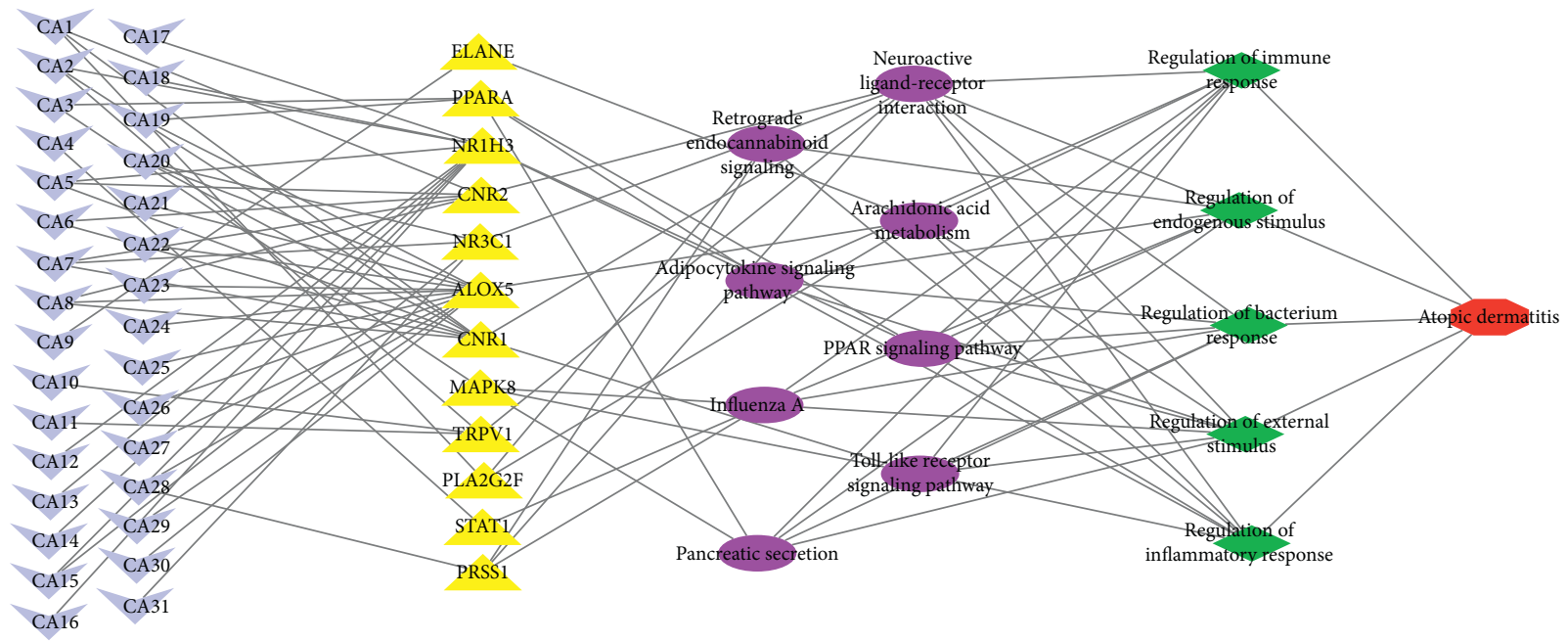

Figure 1: The network of "compound-target-pathway-disease" of Huanglian Jiedu Decoction.

TABLe 3: The levels of IL-4, IL-6, and IL-10 in T lymphocytes in mice with atopic dermatitis.

\begin{tabular}{lcccc}
\hline Group & Concentration $(\mu \mathrm{g} / \mathrm{ml})$ & IL-4 $(\mu \mathrm{g} / \mathrm{mL})$ & IL-6 $(\mu \mathrm{g} / \mathrm{mL})$ & $\mathrm{IL}-10(\mu \mathrm{g} / \mathrm{mL})$ \\
\hline Control group & 0 & $145.14 \pm 12.19$ & $178.42 \pm 18.64$ & $120.98 \pm 15.04$ \\
& 10 & $125.73 \pm 15.31$ & $162.86 \pm 14.13$ & $112.45 \pm 9.77$ \\
Huanglian Jiedu Decoction group & 20 & $90.12 \pm 11.36^{* *}$ & $104.53 \pm 15.02^{* *}$ & $80.27 \pm 14.15^{* *}$ \\
& 40 & $60.08 \pm 9.75^{* *}$ & $78.30 \pm 11.06^{* *}$ & $54.31 \pm 6.99^{* *}$ \\
\hline
\end{tabular}

${ }^{* *} p<0.01$.

\section{Discussion}

Huanglian Jiedu Decoction is the representative prescription with the function of clearing heat and detoxication; by network pharmacological analysis, it is discovered that most of the 81 active ingredients in blood belongs to alkaloid, iridoid, and flavone. The main effective ingredients of Huanglian Jiedu Decoction are berberine, geniposide, and baicalin. Berberine is active in anti-inflammation, and it can suppress proinflammatory reactions by suppressing the signal transfer of mitogen-activated protein kinase and generation of cellular active oxygen [25]; the main ingredient of Scutellaria baicalensis is flavone, and the research of Yang Bo et al. [26] discovered that flavone can bring out the effect of anti-inflammation and immune by the ways of arachidonic acid metabolism, lipoxygenase, and suppressing LTs, cellular factor, and nuclear factor.

The analysis result of network pharmacology shows that the ingredient of Huanglian Jiedu Decoction, such as hericenone $H$, supraene, ethyl oleate (NF), crocetin, and quercetin, can be combined with the proteins of ALOX5, CNR32, NR1H3, CNR1, and NR3C1, and it can affect the synthesis and metabolism of arachidonic acid and reduce the releasing of inflammatory factors such as LTs and cellular factors by many pathways relating to inflammation, such as the arachidonic acid metabolism, PPAR signaling pathway, and Tolllike receptor signaling pathway. Hericenone $\mathrm{H}$ and mandenol can be combined with CNR2, CNR1, and other target proteins and bring out the effect of immune intervention by adjusting Th cell subset (Th1 and Th2Z), and this is closely related to the imbalance of Th 2 and Th1 cell factors during the procedure of atopic dermatitis [5]. The experiment has also proved that Huanglian Jiedu Decoction can indeed reduce the content of IL-4, IL-6, and IL-10 and realize immune intervention by reducing the factors secreted by $\mathrm{T}$ cells.

From the overall analysis of the network of "compoundtarget-pathway-disease" of Huanglian Jiedu Decoction, we can discover that the ingredients of Huanglian Jiedu Decoction can affect many target spots and pathways, and there are also many ingredients affecting one target spot and pathway; all of these present the features of compound preparation of Chinese herbs of multiingredients, multitarget spots, and multipathways. It provides a new researching thought for the research on the effective substances and affecting mechanism of Huanglian Jiedu Decoction treating atopic dermatitis and also provides the necessary rationale for clinical research in future.

\section{Data Availability}

The data used to support the findings of this study are included within this article.

\section{Conflicts of Interest}

The authors declare that they have no conflicts of interest.

\section{Acknowledgments}

This study was supported in part by grants from the National Natural Science Foundation of China (81803681). 


\section{Supplementary Materials}

Information of 81 active components of Huanglian Jiedu Decoction. (Supplementary Materials)

\section{References}

[1] J. J. Lyons, J. D. Milner, and K. D. Stone, "Atopic dermatitis in children," Immunology and Allergy Clinics of North America, vol. 35, no. 1, pp. 161-183, 2015.

[2] R. Vangipuram and S. K. Tyring, "Dupilumab for moderateto-severe atopic dermatitis," Skin Therapy Letter, vol. 22, no. 6, p. 1, 2017.

[3] J. Luo and Z. Song, "Pathogenesis of atopic dermatitis," Chinese Journal of Allergy and Clinical Immunology, vol. 11, no. 04 , pp. 375-381, 2017.

[4] H. Yu and W. Zhang, "Clinical significance of detection of serum total IgE, allergen specific IgE and eosinophils in pati," China Medicine and Pharmacy, vol. 8, no. 1, pp. 136-148, 2018.

[5] K. Kabashima, "New concept of the pathogenesis of atopic dermatitis: interplay among the barrier, allergy, and pruritus as a trinity," Journal of Dermatological Science, vol. 70, no. 1, pp. 3-11, 2013.

[6] W. Chun-Kwok, L. K. Ming-Lam, Q. Huai-Na et al., "Activation of eosinophils interacting with dermal fibroblasts by pruritogenic cytokine IL-31 and alarmin IL-33: implications in atopic dermatitis," PLOS ONE, vol. 7, no. 1, Article ID e29815, 2012.

[7] C. Yung-Sen and C. Bor-Luen, "Mechanism of sleep disturbance in children with atopic dermatitis and the role of the circadian rhythm and melatonin," International Journal of Molecular Sciences, vol. 17, no. 4, pp. 462-472, 2016.

[8] T. Kitamura and Y. Kitamura, "The roles of FOXO1 in various metabolic organs," Seikagaku, vol. 87, no. 2, pp. 176-182, 2015.

[9] M. Leïla, A. Emmanuelle, A.-W. Hélène et al., "TOPICOP?: a new scale evaluating topical corticosteroid phobia among atopic dermatitis outpatients and their parents," PLOS ONE, vol. 8, no. 10, Article ID e76493, 2013.

[10] J. M. Forbes, M. T. Coughlan, and M. E. Cooper, "Oxidative stress as a major culprit in kidney disease in diabetes," $D i$ abetes, vol. 57, no. 6, pp. 1446-1454, 2008.

[11] K.-H. Jeong, T.-W. Lee, C.-G. Ihm, S.-H. Lee, J.-Y. Moon, and S.-J. Lim, "Effects of sildenafil on oxidative and inflammatory injuries of the kidney in streptozotocin-induced diabetic rats," American Journal of Nephrology, vol. 29, no. 3, pp. 274-282, 2009.

[12] Y. Xu, S. Guo, G. Chen et al., "Evaluation of anti-sepsis activity by compounds with high affinity to lipid a from Huanglian Jiedu decoction," Immunopharmacol Immunotoxicol, vol. 39, no. 6, pp. 1-7, 2017.

[13] J. Lu, J.-S. Wang, and L.-Yi Kong, "Anti-inflammatory effects of Huang-Lian-Jie-Du decoction, its two fractions and four typical compounds," Journal of Ethnopharmacology, vol. 134, no. 3, pp. 911-918, 2011.

[14] Y. Sun, M. Ishibashi, T. Seimon et al., "Free cholesterol accumulation in macrophage membranes activates Toll-like receptors and p38 mitogen-activated protein kinase and induces cathepsin K," Circulation Research, vol. 104, no. 4, pp. 455-465, 2009.

[15] H. Zeng, S. Dou, J. Zhao et al., "The inhibitory activities of the components of Huang-Lian-Jie-Du-Tang(HLJDT) on eicosanoid generation via lipoxygenase pathway," Journal of Ethnopharmacology, vol. 135, no. 2011, pp. 561-568, 2010.

[16] Y. Haidan, M. Qianqian, C. Heying et al., "How can synergism of traditional medicines benefit from network pharmacology?" Molecules, vol. 22, no. 7, p. 1135, 2017.

[17] J. Ru, P. Li, J. Wang et al., "TCMSP: a database of systems pharmacology for drug discovery from herbal medicines," Journal of Cheminformatics, vol. 6, no. 1, p. 13, 2014.

[18] D. Gfeller, A. Grosdidier, M. Wirth, A. Daina, O. Michielin, and V. Zoete, "Swiss target prediction: a web server for target prediction of bioactive small molecules," Nucleic Acids Research, vol. 42, no. W1, pp. W32-W38, 2014.

[19] A. P. Davis, T. C. Wiegers, B. L. King et al., "Generating gene ontology-disease inferences to explore mechanisms of human disease at the comparative toxicogenomics database," PLoS ONE, vol. 11, no. 5, Article ID e0155530, 2016.

[20] A. García-Campos Miguel, J. Espinal-Enríquez, and E. Hernández-Lemus, "Pathway analysis: state of the art," Frontiers in Physiology, vol. 6, p. 383, 2015.

[21] M. Chen, F. Ouyang, and S. Zhou, "Adropin as a novel energy factor likely has the ability to regulate blood pressure," Medical Hypotheses, vol. 85, no. 2, pp. 234-239, 2015.

[22] S. Macintyre, L. McKay, and A. Ellaway, "Are rich people or poor people more likely to be ill? Lay perceptions, by social class and neighbourhood, of inequalities in health," Social Science \& Medicine, vol. 60, no. 2, pp. 312-317, 2004.

[23] T. P. O'Connor, "SNAP assay technology," Topics in Companion Animal Medicine, vol. 30, no. 4, pp. 132-138, 2015.

[24] X. Cheng, H. Wang, and X. Wang, "Most probable transition pathways and maximal likely trajectories in a genetic regulatory system," Physica A: Statistical Mechanics and Its Applications, vol. 531, pp. 531-542, 2019.

[25] G. Walsh, "Biopharmaceuticals: recent approvals and likely directions," Trends in Biotechnology, vol. 23, no. 11, pp. 209-214, 2005.

[26] L. E. Zhang, M. Bradley, and L. O. Buxton Iain, "Inositolpolyphosphate binding sites and their likely role in calcium regulation in smooth muscle," International Journal of Biochemistry and Cell Biology, vol. 27, no. 12, pp. 75-81, 1995. 